\title{
PERDA QUÍMICA DE CARBONO E CINÉTICA DO CRESCIMENTO CELULAR EM CULTIVOS DE Spirulina
}

\author{
Michele da Rosa Andrade, Felipe Vieira Camerini e Jorge Alberto Vieira Costa* \\ Escola de Química e Alimentos, Universidade Federal do Rio Grande, CP 474, 96201-900 Rio Grande - RS, Brasil
}

Recebido em 16/12/07; aceito em 12/6/08; publicado na web em 10/11/08

\begin{abstract}
CHEMICAL CARBON LOSSES AND GROWTH KINETICS IN Spirulina CULTURES. Photosynthetic microorganism cultures, such as microalgae, represent one of the alternatives for fossil $\mathrm{CO}_{2}$ emissions mitigation. Carbon supply is the major cost component in microalgal cultures. Aiming to enhance the dissolved inorganic carbon uptake efficiency in microalgal cultures, Spirulina sp LEB18 was cultivated in mediums containing $\mathrm{NaHCO}_{3}$ concentrations ranging from 2.8 to $100 \mathrm{~g} \mathrm{~L}^{-1}$. Results indicated that lower dissolved inorganic carbon concentratios $\left(2.8 \mathrm{~g} \mathrm{~L}^{-1} \mathrm{NaHCO}_{3}\right)$ produce higher growth parameters $\left(\mathrm{X}_{\max }=0.75 \mathrm{~g} \mathrm{~L}^{-1} ; \mathrm{P}_{\max }=0.145 \mathrm{~g} \mathrm{~L}^{-1} \mathrm{~d}^{-1} ; \mu_{\max }=\right.$ $0.254 \mathrm{~d}^{-1}$ ) and lower carbon losses (13.61\%). At $50 \mathrm{~g} \mathrm{~L}^{-1}$ of $\mathrm{NaHCO}_{3}$ cell growth was inhibited and carbon losses reached $38.73 \%$.
\end{abstract}

Keywords: carbon; microalga; Spirulina sp LEB-18.

\section{INTRODUÇÃO}

Microalgas são microrganismos fotossintéticos que podem ser empregados para capturar dióxido de carbono, contribuindo com a redução do efeito estufa no planeta. A biomassa pode ser utilizada como suplemento alimentar ou para a produção de biocombustíveis, entre outras aplicações.

A biomassa microalgal apresenta cerca de $50 \%$ de carbono na sua composição, assim o fornecimento deste nutriente aos cultivos representa um importante componente dos custos de produção, seja gasoso na forma de dióxido de carbono, ou sólido, principalmente na forma de bicarbonato. ${ }^{1}$

A fonte de carbono, uma vez dissolvida no meio de cultivo, participa do equilíbrio químico $\mathrm{CO}_{2(\mathrm{aq})} \leftrightarrow \mathrm{H}_{2} \mathrm{CO}_{3} \leftrightarrow \mathrm{HCO}_{3}^{-} \leftrightarrow \mathrm{CO}_{3}{ }^{2-}$, onde a distribuição entre as espécies químicas é determinada pelo $\mathrm{pH}$. A concentração de sais dissolvidos contribui para a pressão osmótica do meio, que pode ter influência na fisiologia celular da microalga, refletindo nas taxas de crescimento e na composição da biomassa. As cianobactérias geralmente crescem em ambientes alcalinos, onde $\mathrm{HCO}_{3}{ }^{-}$é a forma predominante no equilíbrio químico. ${ }^{2}$

Spirulina é a microalga mais estudada com reconhecida habilidade para fixar carbono inorgânico. ${ }^{3}$ Esta microalga destaca-se por possuir o certificado GRAS (Generally Recognized As Safe) do FDA (Food and Drug Administration), o que garante seu uso como alimento e fármaco. Sua biomassa apresenta alto conteúdo de proteínas (64-74\%), ácidos graxos poliinsaturados, pigmentos e vitaminas. ${ }^{4}$ No entanto, trabalhos voltados ao estudo dos fenômenos associados às reações de equilíbrio do carbono inorgânico dissolvido no meio são ainda escassos.

Assim, o objetivo deste trabalho foi avaliar o crescimento da biomassa e a perda de carbono em cultivos da microalga Spirulina sp LEB-18 em diferentes concentrações de carbono inorgânico dissolvido.

\section{PARTE EXPERIMENTAL}

Foi utilizada a microalga Spirulina sp LEB-18 5 mantida em meio Zarrouk. ${ }^{6}$ A biomassa foi previamente lavada e centrifugada (15 min, $5000 \mathrm{rpm}$ ) para a remoção de sais do meio. A concentração inicial

\footnotetext{
*e-mail: jorgealbertovc@ terra.com.br
}

foi $0,15 \mathrm{~g} \mathrm{~L}^{-1}$, exceto nos ensaios de avaliação da perda de carbono, onde não houve a presença de células.

O meio de cultivo utilizado nos ensaios foi Zarrouk ${ }^{6}$ modificado, onde a fonte de carbono $\left(\mathrm{NaHCO}_{3} 16,8 \mathrm{~g} \mathrm{~L}^{-1}\right)$ foi adicionada em diferentes concentrações, variando entre 2,$8 ; 5 ; 10 ; 20 ; 50 ; 80$ ou $100 \mathrm{~g}$ $\mathrm{L}^{-1}$. O intervalo de concentrações foi definido entre o início do efeito tampão do sal no meio (2,8 $\left.\mathrm{g} \mathrm{L}^{-1}\right)$ até a saturação da solução (100 g $\left.\mathrm{L}^{-1}\right)$. Assim, todos os ensaios iniciaram com o mesmo $\mathrm{pH}(8,2 \pm 0,1)$, eliminando o efeito inicial desta variável no crescimento.

Os experimentos foram descontínuos, realizados em fotobiorreatores do tipo erlenmeyer de $2 \mathrm{~L}$, com $1,8 \mathrm{~L}$ de meio. A temperatura foi mantida em $30{ }^{\circ} \mathrm{C}$ e o fotoperíodo em $12 \mathrm{~h}$ claro/escuro, ${ }^{7}$ com iluminância de 3200 Lux. $^{3}$ A agitação foi realizada através da injeção de ar estéril a 0,3 vvm (volume de ar por volume de meio por min). $\mathrm{O} \mathrm{CO}_{2}$ do ar foi removido por absorção em $\mathrm{NaOH} 2 \mathrm{~N}$.

\section{Determinações analíticas}

A concentração de biomassa de Spirulina sp LEB-18 foi determinada diariamente através da medida do peso seco de uma alíquota de amostra. ${ }^{8} \mathrm{O} \mathrm{pH}$ das culturas foi medido diariamente por $\mathrm{pHmetro}$ digital (Quimis Q.400H, Brasil). A alcalinidade total foi determinada diariamente por titulação do filtrado dos cultivos com $\mathrm{HCl}^{9}{ }^{9}$

\section{Parâmetros de crescimento avaliados}

Ao final de cada ensaio a máxima concentração de biomassa de Spirulina sp LEB-18 ( $\left.\mathrm{X}_{\text {máx }}, \mathrm{g} \mathrm{L}^{-1}\right)$ foi determinada. A produtividade $\left(\mathrm{P}, \mathrm{g} \mathrm{L}^{-1} \mathrm{~d}^{-1}\right)$ foi calculada a partir da equação $\mathrm{P}=\left(\mathrm{X}_{\mathrm{t}}-\mathrm{X}_{0}\right) \cdot\left(\mathrm{t}_{\mathrm{x}}-\mathrm{t}_{0}\right)^{-1}$, onde $\mathrm{X}_{\mathrm{t}}$ é a concentração de biomassa $\left(\mathrm{g} \mathrm{L}^{-1}\right)$ no tempo $\mathrm{t}_{\mathrm{x}}(\mathrm{d})$ e $\mathrm{X}_{0}$ é a concentração de biomassa inicial $\left(\mathrm{g} \mathrm{L}^{-1}\right)$ no tempo $\mathrm{t}_{0}(\mathrm{~d}),{ }^{10}$ sendo a produtividade máxima $\left(\mathrm{P}_{\text {máx }}, \mathrm{g} \mathrm{L}^{-1} \mathrm{~d}^{-1}\right)$ o maior valor de produtividade obtido. A velocidade específica máxima de crescimento $\left(\mu_{\text {máx }}\right.$, $\mathrm{d}^{-1}$ ) foi calculada por regressão exponencial da fase logarítmica da curva de crescimento. ${ }^{11} \mathrm{O}$ conteúdo de carbono inorgânico total dissolvido e a distribuição das espécies químicas no equilíbrio $\mathrm{CO}_{2 \text { (aq) }} \leftrightarrow \mathrm{H}_{2} \mathrm{CO}_{3} \leftrightarrow \mathrm{HCO}_{3}^{-} \leftrightarrow \mathrm{CO}_{3}{ }^{2-}$ foram calculados a partir das frações de ionização, ${ }^{12}$ com valores de $\mathrm{pH}$ e alcalinidade total determinados experimentalmente.

A perda de carbono para a atmosfera foi calculada a partir da equação $\left[\left(\mathrm{C}_{\mathrm{i}}-\mathrm{C}_{\mathrm{f}}\right) *\left(\mathrm{C}_{\mathrm{i}}\right)^{-1}\right]^{*} 100$, onde $\mathrm{Ci}$ é a concentração inicial de 
carbono inorgânico dissolvido no meio e $\mathrm{C}_{\mathrm{f}}$ a concentração final.

\section{RESULTADOS E DISCUSSÃO}

Na Figura 1 são apresentadas as curvas de crescimento de Spirulina sp LEB-18 cultivada em diferentes concentrações de $\mathrm{NaHCO}_{3}$. $\mathrm{O}$ crescimento da biomassa nos ensaios com 5,10 ou $20 \mathrm{~g} \mathrm{~L}^{-1} \mathrm{de}$ $\mathrm{NaHCO}_{3}$ foi semelhante, bem como os parâmetros cinéticos máximos avaliados (Tabela 1), em média $\mathrm{X}_{\text {máx }}=0,6 \mathrm{~g} \mathrm{~L}^{-1}, \mathrm{P}_{\text {máx }}=0,08 \mathrm{~g} \mathrm{~L}^{-1} \mathrm{~d}^{-1} \mathrm{e}$ $\mu_{\text {máx }}=0,13 \mathrm{~d}^{-1}$. Os maiores parâmetros entre todas as concentrações de $\mathrm{NaHCO}_{3}$ testadas ocorreram no ensaio contendo $2,8 \mathrm{~g} \mathrm{~L}^{-1}$ do sal. Neste ensaio a velocidade específica máxima de crescimento $(0,254$ $\left.\mathrm{d}^{-1}\right)$ foi superior à obtida em cultivos de Spirulina com meio Zarrouk padrão, encontrados na literatura $\left(0,093 \mathrm{~d}^{-1},{ }^{13} 0,111 \mathrm{~d}^{-1} ;{ }^{14} 0,2 \mathrm{~d}^{-15}\right)$.

Concentrações de 80 e $100 \mathrm{~g} \mathrm{~L}^{-1}$ de $\mathrm{NaHCO}_{3}$ provocaram inibição do crescimento da microalga (dados não apresentados), enquanto o ensaio com $50 \mathrm{~g} \mathrm{~L}^{-1}$ de $\mathrm{NaHCO}_{3}$ apresentou concentração de biomassa aproximadamente constante durante o cultivo (Figura 1).

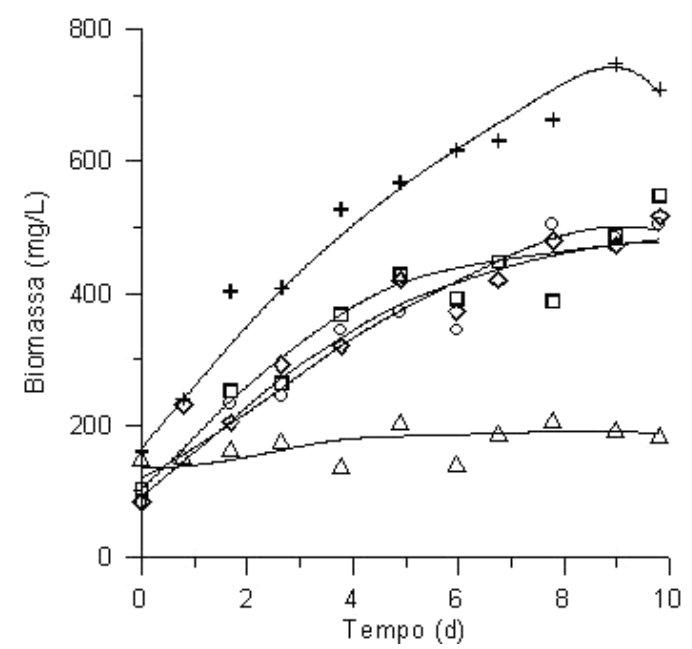

Figura 1. Variação da biomassa de Spirulina sp LEB-18 em diferentes concentrações de $\mathrm{NaHCO}_{3}$ em $g L^{-1}:(+) 2,8 ;(\diamond) 5 ;(\square) 10 ;(\mathrm{O}) 20 ;(\triangle) 50$

Tabela 1. Parâmetros cinéticos da microalga Spirulina sp LEB-18 em diferentes concentrações de $\mathrm{NaHCO}_{3}$. Concentração máxima de biomassa $\left(\mathrm{X}_{\text {máx }}, \mathrm{g} \mathrm{L}^{-1}\right)$; Produtividade máxima $\left(\mathrm{g} \mathrm{L}^{-1} \mathrm{~d}^{-1}\right)$; Velocidade específica máxima de crescimento $\left(\mu_{\text {máx }}, d^{-1}\right)$

\begin{tabular}{lcccc}
\hline $\begin{array}{l}\mathrm{NaHCO}_{3} \\
\left(\mathrm{~g} \mathrm{~L}^{-1}\right)\end{array}$ & $\mathrm{P}_{\text {máx }}$ & $\mathrm{X}_{\text {máx }}$ & $\mu_{\text {máx }}$ & $\mathrm{R}^{2 *}$ \\
\hline 2,8 & 0,145 & 0,75 & 0,25 & 0,9910 \\
5,0 & 0,079 & 0,64 & 0,12 & 0,9987 \\
10,0 & 0,088 & 0,55 & 0,16 & 0,9978 \\
20,0 & 0,069 & 0,50 & 0,10 & 0,9997 \\
50,0 & 0,011 & 0,21 & 0,09 & 0,9975 \\
\hline
\end{tabular}

* Coeficiente de regressão exponencial da fase logarítmica de crescimento

Quando as células são expostas à alta concentração de $\mathrm{NaHCO}_{3}$ a fotossíntese pode ser inibida, devido à rápida entrada de sódio na célula. ${ }^{3} \mathrm{~A}$ atividade fotossintética pode também ser inibida devido ao alto teor de carbonato $\left(\mathrm{CO}_{3}{ }^{2-}\right)$ formado. ${ }^{16,17}$ No ensaio com $50 \mathrm{~g} \mathrm{~L}^{-1}$ de $\mathrm{NaHCO}_{3}$, a concentração de $\mathrm{CO}_{3}{ }^{2-}$ alcançou $6,3 \mathrm{~g} \mathrm{~L}^{-1}$ e o pH 10,1 (Figura 2a). O lento crescimento celular neste ensaio com $50 \mathrm{~g} \mathrm{~L}^{-1}$ de $\mathrm{NaHCO}_{3}$ pode ainda estar associado com o aumento da energia requerida para expulsar o íon $\mathrm{Na}^{+}$da célula, provocando elevadas taxas de respiração noturna, que consome parte da biomassa formada no período claro. ${ }^{3,8}$

\section{Perda de carbono nos cultivos}

Devido à natureza apolar da membrana plasmática, a resistência à difusão da molécula de $\mathrm{CO}_{2}$ é menor que a do íon $\mathrm{HCO}_{3}^{-}$, tornando o $\mathrm{CO}_{2}$ a espécie química de carbono inorgânico preferencialmente consumida pelas microalgas. ${ }^{17}$ Quando este é consumido, o sistema em equilíbrio $\mathrm{CO}_{2(\text { aq) }} \leftrightarrow \mathrm{H}_{2} \mathrm{CO}_{3} \leftrightarrow \mathrm{HCO}_{3}^{-} \leftrightarrow \mathrm{CO}_{3}{ }^{2-}$ reage no sentido de formação de $\mathrm{CO}_{2}$, consumindo $\mathrm{H}^{+}$do meio. Por outro lado, quando a concentração de $\mathrm{HCO}_{3}{ }^{-}$é alta, o consumo desta espécie química pode ocorrer pelo seu transporte ativo através da membrana plasmática ${ }^{18}$ ou por sua conversão a $\mathrm{CO}_{2}$ pela enzima anidrase carbônica, segundo a reação $\mathrm{HCO}_{3}^{-} \leftrightarrow \mathrm{CO}_{2}+\mathrm{OH}^{-} \cdot{ }^{3,19,20}$ Assim, seja $\mathrm{CO}_{2}$ ou $\mathrm{HCO}_{3}^{-}$a espécie química consumida pelas células, o crescimento celular resulta em aumento do $\mathrm{pH}$ do meio de cultivo.

Nos ensaios com 50, 80 e $100 \mathrm{~g} \mathrm{~L}^{-1}$ de $\mathrm{NaHCO}_{3}$ o crescimento da biomassa foi inibido. No entanto, ocorreu aumento do $\mathrm{pH}$ (Figura 2a) e queda da concentração de íons $\mathrm{HCO}_{3}^{-}$, indicando que, além do crescimento da biomassa, outros fatores provocam a alteração do $\mathrm{pH}$
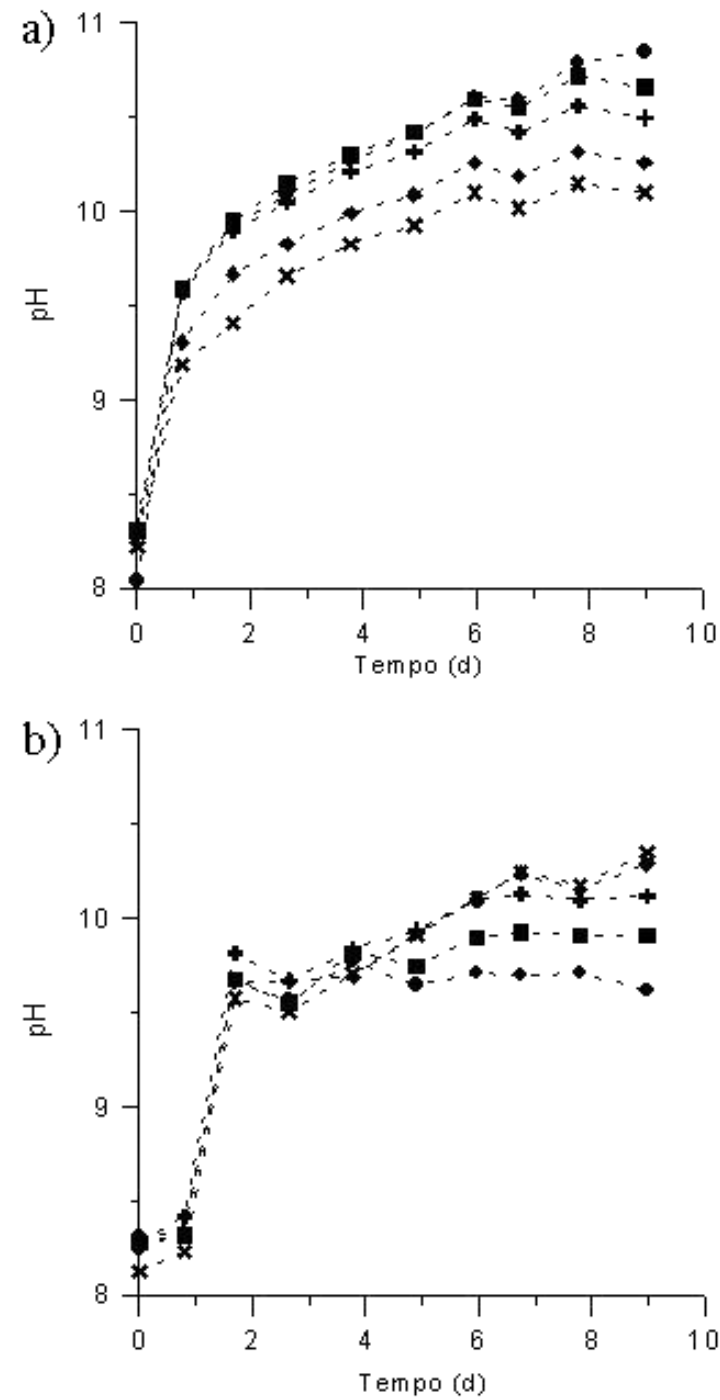

Figura 2. Variação do pH do meio Zarrouk ao longo do tempo em diferentes

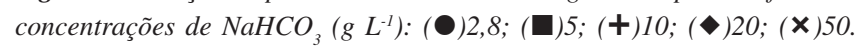
Meio com células (a) e sem células (b) 
e da concentração de carbono inorgânico dissolvido no meio durante o cultivo de microalgas.

Soluções ricas em carbono inorgânico dissolvido, como o meio Zarrouk, contêm $\mathrm{CO}_{2}$ livre em concentração superior $\left(0,05 \mathrm{~g} \mathrm{~L}^{-1}\right)$ à de equilíbrio com a atmosfera $\left(0,0004 \mathrm{~g} \mathrm{~L}^{-1} \mathrm{CO}_{2}\right.$ a $\left.30{ }^{\circ} \mathrm{C}\right) .{ }^{21}$ Enquanto houver esse gradiente, ocorre um fluxo de $\mathrm{CO}_{2}$ do meio de cultivo para a atmosfera.

$\mathrm{O}$ deslocamento do equilíbrio $\mathrm{CO}_{2(\mathrm{aq})} \leftrightarrow \mathrm{H}_{2} \mathrm{CO}_{3} \leftrightarrow \mathrm{HCO}_{3}^{-} \leftrightarrow$ $\mathrm{CO}_{3}{ }^{2-}$ no sentido da reposição do $\mathrm{CO}_{2}$ perdido, com conseqüente consumo de $\mathrm{H}^{+}$, provoca o aumento do $\mathrm{pH}$ do meio de cultivo até que a concentração de $\mathrm{CO}_{2}$ dissolvido no meio líquido esteja em equilíbrio com a concentração de $\mathrm{CO}_{2}$ na atmosfera.

O fluxo de massa entre o meio de cultivo e a atmosfera é proporcional ao gradiente de concentração entre as duas fases. ${ }^{22}$ Considerando que a concentração de $\mathrm{CO}_{2}$ livre no meio aumenta em função do aumento da concentração total de carbono inorgânico dissolvido, a maior perda de $\mathrm{CO}_{2}$ e a maior variação de $\mathrm{pH}$ são verificadas nos meios com as maiores concentrações de $\mathrm{NaHCO}_{3}$ (Figura 2b).

A Figura 3 mostra a queda na concentração de $\mathrm{CO}_{2}$ nos meios na ausência de Spirulina sp LEB-18. A partir do segundo dia de cultivo a concentração de $\mathrm{CO}_{2}$ livre tornou-se aproximadamente igual e constante em todas as concentrações de $\mathrm{NaHCO}_{3}$ testadas. Assim, a utilização de meios sintéticos com altas concentrações de carbono inorgânico no cultivo de microalgas deve ser evitada, uma vez que sua composição inicial é alterada em função das perdas de carbono para o meio externo, resultando em um meio com uma concentração de $\mathrm{CO}_{2}$ dissolvido próxima à de equilíbrio com a atmosfera.

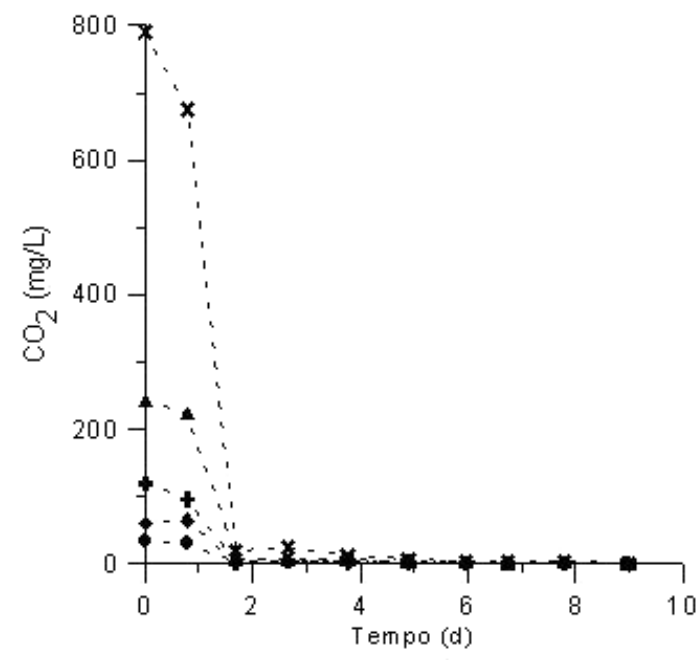

Figura 3. Concentração de $\mathrm{CO}_{2}$ em ensaios na ausência de células de Spirulina sp LEB-18 contendo diferentes concentrações de $\mathrm{NaHCO}_{3}\left(g \mathrm{~L}^{-1}\right):(\mathbf{O})$ 2,8; ( ) $5 ;(\mathbf{+}) 10 ;(\mathbf{\Delta}) 20 ;(\mathbf{X}) 50$

No ensaio contendo $2,8 \mathrm{~g} \mathrm{~L}^{-1}$ de $\mathrm{NaHCO}_{3}$, onde foram verificados os melhores parâmetros de crescimento para a microalga entre todas as concentrações de $\mathrm{NaHCO}_{3}$ testadas (Tabela 1), foi verificada a maior diferença entre as variações de $\mathrm{pH}$ provocadas pela perda de $\mathrm{CO}_{2}$ para a atmosfera e as variações provocadas pelas perdas somadas ao consumo biológico (Figura 4a). A Figura 4b mostra a variação da concentração de $\mathrm{NaHCO}_{3}$ neste ensaio, evidenciando a diferença entre o consumo biológico de carbono e as perdas para a atmosfera. As perdas de carbono em função da concentração de $\mathrm{NaHCO}_{3}$ no meio são apresentadas na Figura 5, demonstrando que o aumento na concentração de carbono inorgânico dissolvido utilizado no preparo do meio Zarrouk provoca maiores perdas de carbono para a atmosfera.

Além das perdas de carbono dos cultivos para a atmosfera
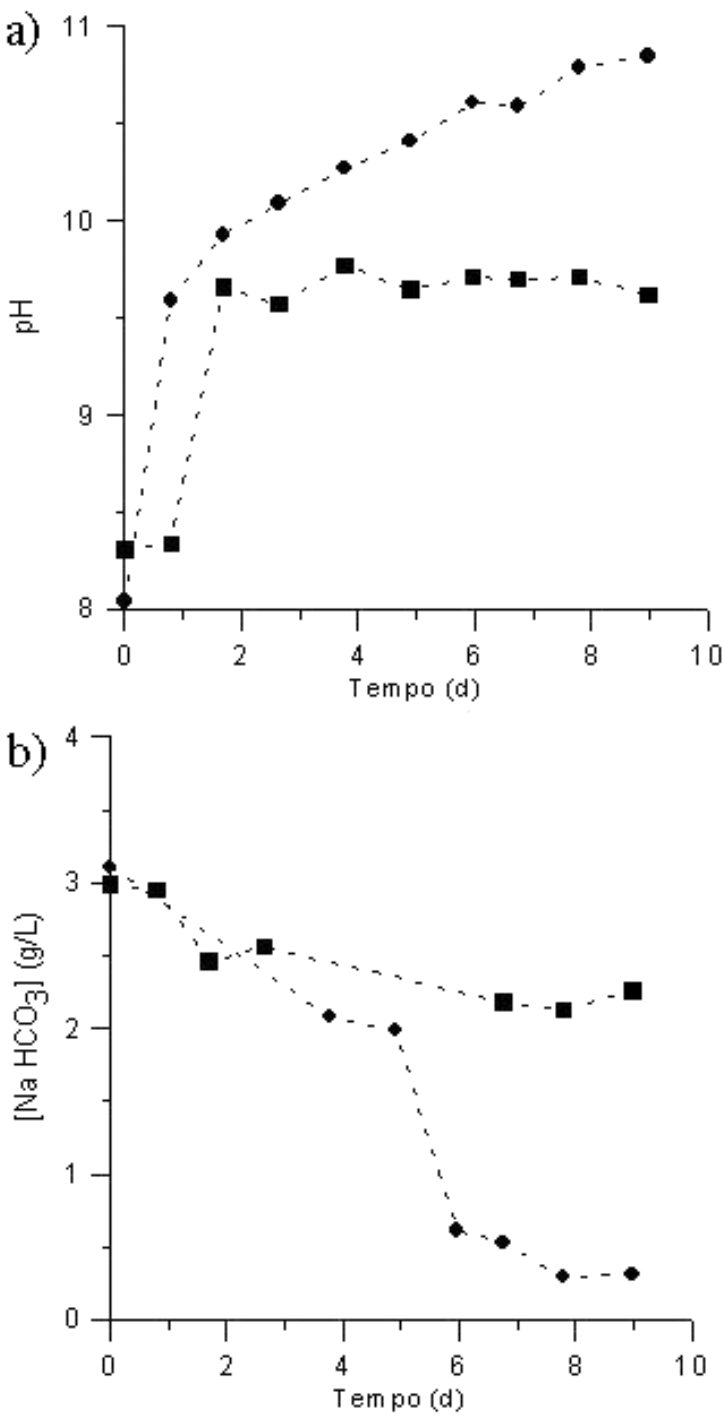

Figura 4. Variação do $\mathrm{pH}$ (a) e da concentração de $\mathrm{NaHCO}_{3}$ em $\mathrm{g} \mathrm{L}^{-1}$ (b) no ensaio contendo 2,8 $\mathrm{g} \mathrm{L}^{-1}$ de $\mathrm{NaHCO}_{3}$. Meio com células (-) e sem células (ם)

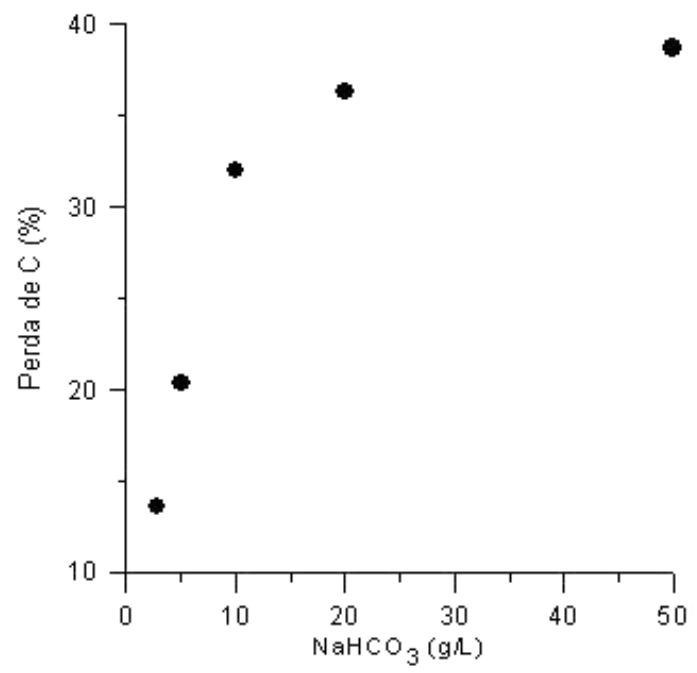

Figura 5. Perdas de carbono inorgânico dissolvido em função da concentração inicial de $\mathrm{NaHCO}_{3}$ no meio de cultivo 
representarem um custo adicional na produção de biomassa, o aumento de $\mathrm{pH}$ associado pode conduzir a valores de $\mathrm{pH}$ inibitórios ao crescimento celular. Poucos autores consideram as perdas químicas de carbono nos cultivos, de modo que em estudos sobre o $\mathrm{pH}$ e o consumo de carbono em cultivos de microalgas estas perdas podem ser confundidas com o consumo biológico de carbono pelas células, levando a resultados equivocados. ${ }^{21,23,24}$

A elevação do $\mathrm{pH}$ do meio de cultivo reduz a concentração de $\mathrm{CO}_{2}$ livre, segundo o equilíbrio, dificultando a perda química deste componente para a atmosfera, além de reduzir a suscetibilidade do cultivo de Spirulina à contaminação. ${ }^{25}$ Assim, microalgas alcalifílicas como Spirulina apresentam características biológicas adequadas às condições físico-químicas do meio, que levam à maior eficiência do consumo de carbono.

\section{CONCLUSÃO}

A menor perda química de $\mathrm{CO}_{2}$ para a atmosfera $(13,61 \%)$ e os maiores parâmetros de crescimento $\left(\mathrm{X}=0,75 \mathrm{~g} \mathrm{~L}^{-1}, \mathrm{P}=0,145 \mathrm{~g} \mathrm{~L}^{-1}\right.$ $\left.\mathrm{d}^{-1}, \mu=0,254 \mathrm{~d}^{-1}\right)$ da microalga Spirulina sp LEB-18 ocorreram no ensaio com a menor concentração de carbono testada $\left(\mathrm{NaHCO}_{3} 2,8 \mathrm{~g}\right.$ $\mathrm{L}^{-1}$ ). Com o aumento da concentração de bicarbonato os parâmetros de crescimento diminuíram e as perdas de carbono foram maiores. Em $50 \mathrm{~g} \mathrm{~L}^{-1}$ de $\mathrm{NaHCO}_{3} 38,73 \%$ do carbono do meio foi perdido para a atmosfera, além de ocorrer inibição do crescimento celular.

A manutenção dos cultivos em baixas concentrações de carbono inorgânico dissolvido pode resultar em maior eficiência no consumo do nutriente pela microalga Spirulina sp LEB-18, contribuindo para a redução de custos em plantas de produção da biomassa microalgal.

\section{REFERENCIAS}

1. Vonshak, A. Em Spirulina platensis (Arthrospira) Physiology, cellbiology and biotechnology; Vonshak, A., ed.; Taylor \& Francis: London, 1997.

2. Richmond, A. Em Handbook of Algal Mass Culture; Richmond, A., ed.; CRC Press: USA, 1986.
3. Morais, M. G.; Costa, J. A. V.; J. Biotechnol. 2007, 129, 439.

4. Cohen, Z. Em ref. 1.

5. Morais, M. G.; Costa, J. A. V.; Biotechnol. Lett. 2007, 29, 1349.

6. Zarrouk, C.; Tese de Doutorado, University of Paris, Paris, 1966.

7. Costa, J. A. V.; Colla, L. M.; Duarte Filho, P. D.; Kabke, K.; Weber, A.; World J. Microbiol. Biotechnol. 2002, 18, 1.

8. Torzillo, G.; Sacchi, A.; Materasi, R.; Bioresour. Technol. 1991, 38, 95.

9. Clesceri, L. S.; Greenberg, A. E.; Eaton, A. D.; Standard Methods for the Examination of Water and Wastewater, $19^{\text {th }}$ ed., Amer. Publ. Health Ass.: Washington, 1998.

10. Schmidell, W.; Lima, A. U.; Aquarone, E.; Borzani, W.; Biotecnologia Industrial, Edgard Blücher Ltda.: São Paulo, 2001, vol. 2.

11. Bailey, J. E.; Ollis, D. F.; Biochemical Engineering Fundamentals, $2^{\text {nd }}$ ed., McGraw-Hill: Singapore, 1986.

12. Carmouze, J. P.; O Metabolismo dos Ecossistemas Aquáticos: Fundamentos teóricos, métodos de estudo e análises químicas, Edgard Blücher Ltda.: São Paulo, 1994.

13. Andrade, M. R.; Costa, J. A. V.; Aquaculture 2007, 264, 130.

14. Reichert, C. C.; Reinehr, C. O.; Costa, J. A. V.; Braz. J. Chem. Eng. 2006, 23, 23.

15. Costa, J. A. V.; Linde, G. A.; Atala, D. I. P.; World J. Microbiol. Biotechnol. 2000, 16, 15.

16. Binaghi, L.; Borghi, A. D.; Lodi, A.; Converti, A.; Borghi, M. D.; Process Biochem. 2003, 38, 1341.

17. Miller, A. G.; Colman, B.; Plant. Physiol. 1980, 65, 397.

18. James, P. L.; Larkum, A. W. D.; Aquat. Bot. 1996, 55, 149.

19. Shiraiwa, Y.; Goyal, A.; Tolbert, N. E.; Plant Cell Physiol. 1993, 34, 649.

20. Badger, M. R.; Price, G. D.; Physiol. Plant. 1992, 84, 606.

21. Alava, D.; Mello, P. C.; Wagener, K.; J. Braz. Chem. Soc. 1997, 8, 447.

22. Fick, A.; Philos. Mag. 1855, 10, 30.

23. Maroto, J. M. R.; Jimenez, C.; Aguilera, J.; Niell, F. X.; Aquac. Eng. 2005, 32, 493.

24. Livansky, K.; Pilarski, P. S.; Algol. Stud. 1993, 97, 113.

25. Richmond, A.; Grobbelaar, J. U.; Biomass 1986, 10, 253. 